\title{
Neural Connectivity and Neuropsychological Function
}

\author{
Edith V. Sullivan
}

Received: 5 April 2010 /Accepted: 29 April 2010 / Published online: 22 May 2010

(C) Springer Science+Business Media, LLC 2010

A tradition in neuropsychology has been to devise tests that are sensitive and selective to lesions in specific brain structures. The structures supporting the functions have been implicitly understood to be gray matter-neurons, nuclei-the seat of function. One-to-one, brain-to-behavior relations between a neuronal locus and a single function were the gold standard of early neuropsychologists and founded on "pure" cases with circumscribed, single lesions. This view of brain-behavior relations proved to be essential for initial attempts to establish neural substrates of cognitive, sensory, and motor functions and for convincing the scientific and medical communities of the functional selectivity of brain sites. Despite the importance of these neuro/psychological concepts, they ultimately have proved to be unrealistically narrow to account for many, if not most, patterns of functional impairments. In the majority of individuals who sustain brain insult, the damage is commonly multi-focal, whether from dementing disorders, closed head injury, or even focal hippocampal epilepsy, a concomitant being bilateral extrahippocampal tissue volume deficits. The observations and insights of Norman Geschwind laid the groundwork for breaking set from the purists' accounts of brain structure-function relations and for providing a brain-based framework for interpreting impairments resulting from multi-focal lesions.

Geschwind's two-part treatise on "Disconnexion Syndromes in Animals and Man" presented explicit instantiations of how his version of disconnectionism could realistically account for "islands" of function cut off from the rest of the brain. Geschwind wrote, "...disconnexion of

\section{E. V. Sullivan $(\triangle)$}

Department of Psychiatry and Behavioral Sciences,

Stanford University School of Medicine,

Stanford, CA 94305-5723, USA

e-mail: edie@stanford.edu cortical regions can be achieved by lesions involving either white matter connexions or by damage to association areas which constitute obligatory way stations between the primary sensory, motor, and limbic regions..." (Geschwind 1965a) (page 290). This is a main tenet of his expansive work.

In this issue of Neuropsychology Review, we have the privilege of reprinting Part I of Geschwind's influential arguments for a disconnection basis of neuropsychological dysfunction of many neuropsychiatric disorders affecting the CNS. Readers of Part I will surely be enticed to seek out Part II (Geschwind 1965b), which provides in-depth analysis of the agnosias, apraxias, aphasias, consciousness, and relevant historical background lending further support to the disconnection concept. In his literature review, Geschwind himself recognized that his ideas were not entirely novel. His contribution, nonetheless, was the assemblage and integration of historical accounts with his own empirical observations and lucid presentation of elusive concepts of the burgeoning field of neuropsychology.

In addition to the reprinting of the Geschwind paper, this issue of Neuropsychology Review presents a commentary, retrospective, and four new reviews, each describing numerous instances and broad uses of the disconnection model.

Bruce Miller provides a cogent review of Geschwind's contribution from a behavioral neurologist's perspective.

Michael Geschwind, also a behavioral neurologist and relative of Normal Geschwind, presents his personal recounting of his unexpected professional rendezvous with his cousin's concepts.

Christopher Filley focuses on the functional relevance of white matter pathology identified with neuroimaging in neurological dementing and degenerative disorders. A second focus is cerebral white matter disorders, often underappreciated as substrates of selective neuropsychological impairment associated with each disorder. 
Fig. 1 Midsagittal view of a diffusion tensor image $(D T I)$ of fractional anisotropy in gray tones, where brighter intensities in white matter reflect more highly and linearly organized microstructure. Superimposed are three-dimensional, bilateral depictions of fiber bundles identified with fiber tracking of DTI data: mustard $=$ superior cingulate bundle; green $=$ inferior cingulate bundle; blue $=$ corticospinal tracts; orange $=$ fornix; red=pontocerebellar tracts. Image courtesy Adolf Pfefferbaum, M.D., Director, Neuroscience Program, SRI International and Professor Emeritus, Stanford University School of Medicine

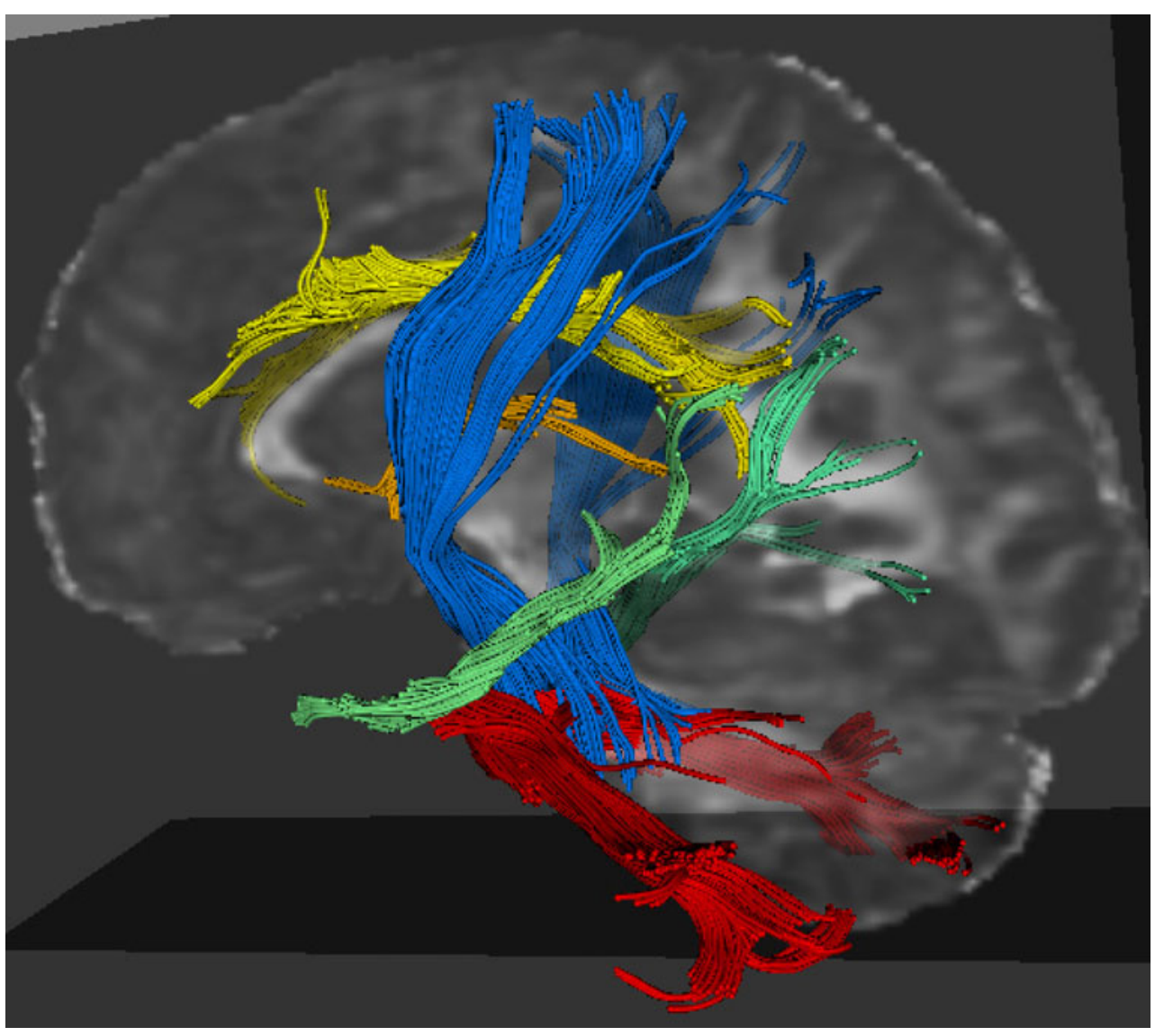

Tilman Schulte and Eva Müller-Oehring delineate the role of the corpus callosum and its anatomical and functional divisions in interhemispheric integration of visuomotor and cognitive processes. The authors present exemplary studies based on quantitative measurement of regional callosal integrity determined with structural MRI, DTI, and functional MRI in conjunction with development and application of creative tests of callosal integrity.

Alice Cronin-Golomb takes a unique view of the neuropsychological sequelae of Parkinson's disease as a disconnection syndrome. This creative application of the disconnection model should be influential in understanding other neurological degenerative diseases.

Sandra Chanraud, Natalie Zahr, Adolf Pfefferbaum and I present a brief overview of the enormously growing field of MR diffusion tensor imaging (DTI) and its application in neuropsychology. The visual depiction (Fig. 1) and quantification of mathematically-modeled fiber tracts produced with DTI have provided unprecedented evidence for the relevance of white matter disruption - even when not a complete lesion - to impairment in selective neuropsychological function. We can only imagine how Norman Geschwind would have enjoyed challenging his model with this imaging technology.
A May 2010 search on "diffusion tensor imaging" in PubMed alone yielded 3537 articles published since its earliest applications in 1994. This burst of work indicates the importance of taking account of the condition of white matter microstructure and its effect on neural systems communication. It also indicates the need for well-founded models for hypothesis testing and data interpretation. Indeed, efforts to interpret neuroradiological evidence for degraded fiber integrity in conditions affecting brain white matter commonly invoke Geschwind's disconnection model. This issue of Neuropsychology Review provides the opportunity to those who exploit the model to read his primary work.

Acknowledgment This work was supported by grants from the NIAAA (AA010723, AA017168, AA017923) and NIA (AG017919).

Disclosures The author is the editor-in-chief of the journal.

\section{References}

Geschwind, N. (1965a). Disconnexion syndromes in animals and man. I. Brain, 88, 237-294.

Geschwind, N. (1965b). Disconnexion syndromes in animals and man. II. Brain, 88, 585-644. 